ARTICLE

\title{
Machine learning-guided acyl-ACP reductase engineering for improved in vivo fatty alcohol production
}

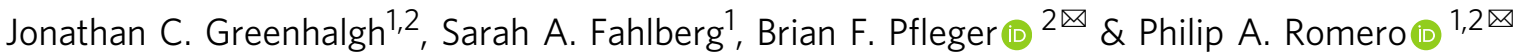

\begin{abstract}
Alcohol-forming fatty acyl reductases (FARs) catalyze the reduction of thioesters to alcohols and are key enzymes for microbial production of fatty alcohols. Many metabolic engineering strategies utilize FARs to produce fatty alcohols from intracellular acyl-CoA and acyl-ACP pools; however, enzyme activity, especially on acyl-ACPs, remains a significant bottleneck to high-flux production. Here, we engineer FARs with enhanced activity on acyl-ACP substrates by implementing a machine learning $(\mathrm{ML})$-driven approach to iteratively search the protein fitness landscape. Over the course of ten design-test-learn rounds, we engineer enzymes that produce over twofold more fatty alcohols than the starting natural sequences. We characterize the top sequence and show that it has an enhanced catalytic rate on palmitoyl-ACP. Finally, we analyze the sequence-function data to identify features, like the net charge near the substrate-binding site, that correlate with in vivo activity. This work demonstrates the power of ML to navigate the fitness landscape of traditionally difficult-to-engineer proteins.
\end{abstract}

\footnotetext{
${ }^{1}$ Department of Biochemistry, University of Wisconsin-Madison, Madison, WI, USA. ${ }^{2}$ Department of Chemical \& Biological Engineering, University of

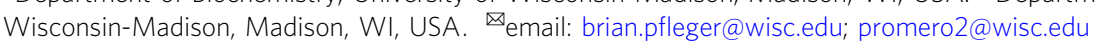


F atty acyl reductases (FARs) are vital for the microbial synthesis of key primary and secondary metabolites such as fatty aldehydes, waxes, alkanes, and fatty alcohols. These enzymes often interface with fatty acid anabolic/catabolic pathways and catalyze the reduction of thioester bonds found in acylacyl carrier proteins ${ }^{1}$ (acyl-ACPs) and acyl-coenzyme A's (acyl-CoAs) ${ }^{2}$. These enzymes typically have a preference for either acyl-ACP or acyl-CoA substrates, but also display cross reactivity due to the common thioester bond in both substrates. Some FARs perform only one, two-electron, reduction step to produce aldehydes ${ }^{3}$, while others can perform two sequential reduction steps (totaling four electrons) to produce alcohols directly ${ }^{4-6}$.

The alcohol-forming FAR enzymes capable of complete reduction of thioesters to alcohols have been widely used in metabolic engineering for producing fatty alcohols ${ }^{7-11}$. The enzymes Maqu 2220 and MA-ACR from Marinobacter aquaeloei display high activity on acyl-CoA substrates and produce the corresponding fatty alcohols $2,4,11$. These enzymes can be incorporated to feed off of the reverse beta oxidation pathway to yield high levels of alcohols ${ }^{8}$. Another common metabolic engineering strategy involves terminating the host organism's fatty acid elongation cycle with a thioesterase to produce a fatty acid that can then be converted to an acyl-CoA by an ATP-dependent ligase, and then finally converted to an alcohol by a $\mathrm{FAR}^{7,9,12}$. This approach was recently applied using an engineered C8specific thioesterase to produce octanol at a titer of $1.3 \mathrm{~g} / \mathrm{L}^{9}$. While these titers are impressive, alcohol production could be more efficient with enzymes that bypass the thioesterase-ligase route, and instead directly convert acyl-ACPs to alcohols ${ }^{13}$.

Alcohol-forming FARs that prefer acyl-ACP substrates are less well characterized, and often display low to moderate activity relative to enzymes that prefer acyl-CoA substrates. Engineering alcohol-forming FARs such as MA-ACR to have higher activity on acyl-ACP substrates would open up new highly efficient pathways to making fatty alcohols in vivo. However, these enzymes are challenging to engineer using traditional protein engineering methods. MA-ACR and its close homologs lack highresolution crystal structures needed for most computational and rational engineering approaches. Directed evolution strategies are also difficult because fatty alcohol production cannot be assayed in high-throughput. Machine learning (ML)-based protein engineering has recently emerged as an efficient strategy for engineering proteins with limited structural and functional information ${ }^{14-20}$. Machine learning algorithms can infer the protein sequence-function mapping given a limited experimental sampling of the landscape ${ }^{14}$. The resulting sequence-function models can be used to computationally explore sequence space and predict optimized sequences.

In this work, we apply an ML-based protein engineering framework to engineer acyl-ACP reductases to produce fatty alcohols in vivo. We start by characterizing the ability of MA-ACR and related enzymes to produce fatty alcohols from intracellular acyl-ACP pools. We then design a large library of chimeric enzymes and develop an ML-based protein optimization strategy to rapidly identify highly active sequences. Our approach consists of generating diverse initial sequence sampling to get a preliminary view of the landscape, followed by ten iterative designtest-learn cycles to efficiently search the landscape and discover optimized sequences. We show that the algorithm converges on highly active acyl-ACP reductases that produce 4.9-fold more fatty alcohols than MA-ACR. We evaluate the performance of the engineered enzymes in vitro and find the improved alcohol titers are the result of engineered enzymes with increased catalytic efficiency. Finally, we perform a statistical analysis of the landscape and identify key sequence elements that contribute to enzyme activity. Many of these elements are located near the enzyme's putative substrate entry channel and may be involved with modulating the preference between acyl-CoA and acyl-ACP substrates. These results open future directions to engineer enzymes for efficient microbial production of fatty alcohols.

\section{Results}

In vivo fatty alcohol production by natural and chimeric acylACP reductases. We focused our protein engineering efforts on MA-ACR from Marinobacter aquaeloei because it displays high in vivo activity on acyl-CoA substrates ${ }^{7-9}$, and it was also suspected to accept acyl-ACP substrates. MA-ACR consists of two domains that sequentially reduce thioesters to alcohols (Fig. 1a). The C-terminal acyl-thioester reductase (ATR) domain reduces thioesters from ACP or CoA substrates to aldehydes, and the $\mathrm{N}$-terminal aldehyde reductase domain (AHR) reduces aldehydes to alcohols ${ }^{4}$. We also identified two related enzymes from Marinobacter BSs 20148 and Methylibium Sp. T29 that have 60-81\% sequence identity with MA-ACR (Fig. 1b) and were previously shown to produce alcohols from acyl-CoAs $s^{8,9}$. Throughout the remainder of this paper, we refer to the FAR enzymes from Marinobacter aquaeloei, Marinobacter BSs20148, and Methylibium Sp. T29 as MA-ACR, MB-ACR, and MT-ACR, respectively.

We characterized the ability of these three natural enzymes to produce fatty alcohols from intracellular acyl-ACP pools by introducing them into E. coli RL08ara ${ }^{21}$, a strain that lacks the $f a d D$ gene, which encodes an acyl-CoA ligase. Deletion of fadD decreases the formation of acyl-CoAs and thus presents the enzymes with substrates that are predominantly acyl-ACPs from fatty acid biosynthesis ${ }^{10,13}$. We grew each strain under aerobic conditions, extracted the fatty alcohols and measured the fatty alcohol (C6-C16) titers using gas chromatography. We found the enzyme MB-ACR from Marinobacter BSs20148 displayed more than double the total fatty alcohol titer of MA-ACR (Fig. 1c). These results suggest that $\mathrm{MB}-\mathrm{ACR}$ may have a preference for acyl-ACP substrates because it was previously shown to have lower activity than MA-ACR on acyl-CoA substrates ${ }^{8}$.

We next characterized the fatty alcohol production from chimeric enzymes generated by swapping AHR and ATR domains between the three natural sequences. Of the six possible chimeric enzymes, we found the chimera with an AHR domain from MA-ACR and the ATR domain from MB-ACR displayed the highest fatty alcohol titers (Fig. 1c). This chimeric enzyme produced $\sim 50 \%$ more fatty alcohol than MB-ACR and roughly three-fold more fatty alcohol than MA-ACR. The ATR domain from MT-ACR also displayed increased activity $(\sim 1.5 \mathrm{x})$ when fused to the AHR domain from MA-ACR. These results suggest that MA-ACR's AHR domain is more efficient than the AHR domains from the two other natural enzymes.

To further explore how gene shuffling can enhance fatty alcohol production, we designed a large library of ATR domains using SCHEMA ${ }^{22-24}$ structure-guided recombination (Fig. 1d). Our design used a homology model of MA-ACR's ATR domain to define the family's contact map and identified seven breakpoints within the domain that balance structural disruption with library diversity (Supplementary Fig. 1). These seven breakpoints define eight sequence blocks that span the ATR domain's structure (Fig. 1e). Notably, the structure's substrate access channel is composed of blocks $4,5,6,7$, and 8 , and diversity at these positions may result in changes in the enzyme's substrate preference. Each of the eight sequence blocks can be inherited from one of the three natural enzymes to define a combinatorial sequence space of $3^{8}$ sequences. However, block 6 from MA-ACR and MB-ACR happened to be perfectly conserved, and therefore the total library diversity is $2^{*} 3^{7}=4374$ sequences. We fused our 
a

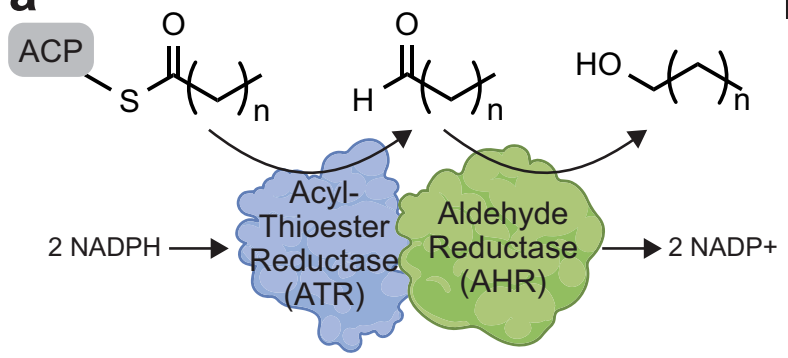

b

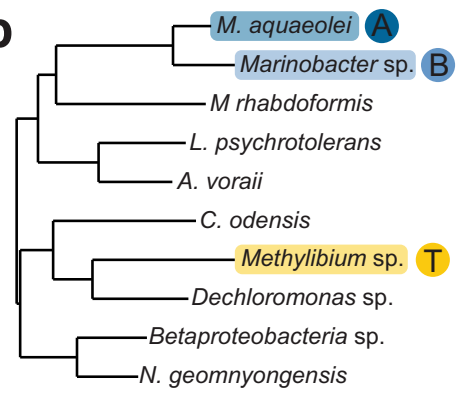

C

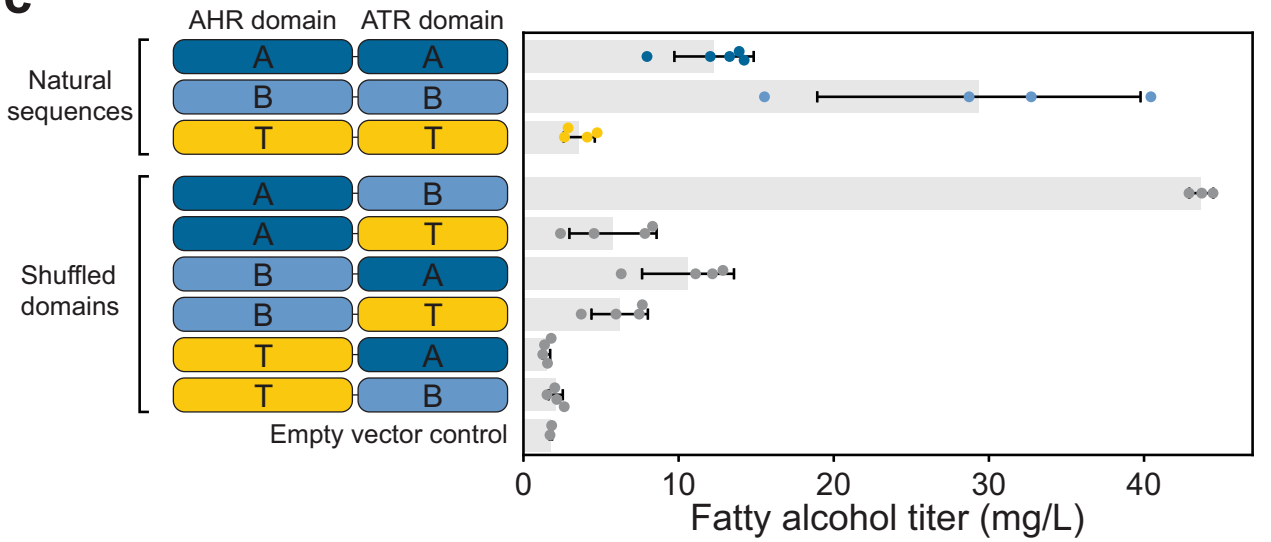

d

SCHEMA recombination of ATR domain

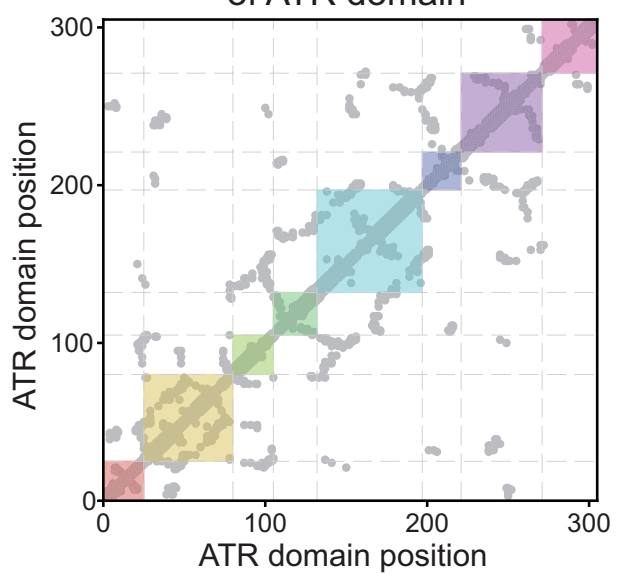

e

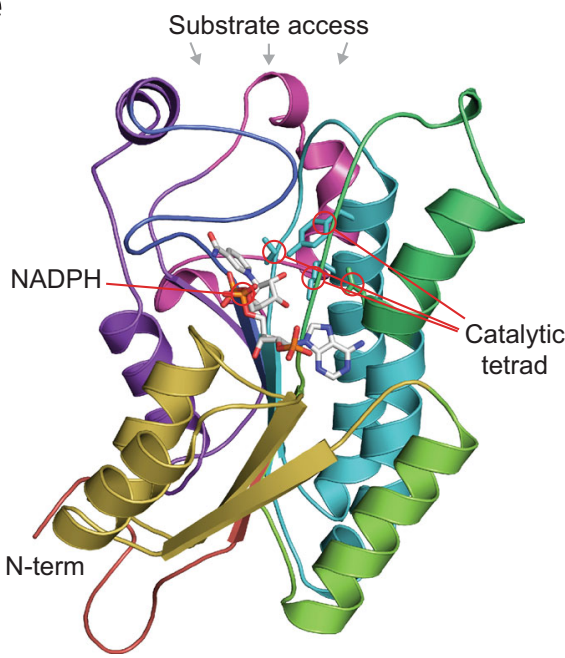

f Chimeric enzyme library

AHR domain from MA-ACR (A) Eight SCHEMA blocks from A, B, or T

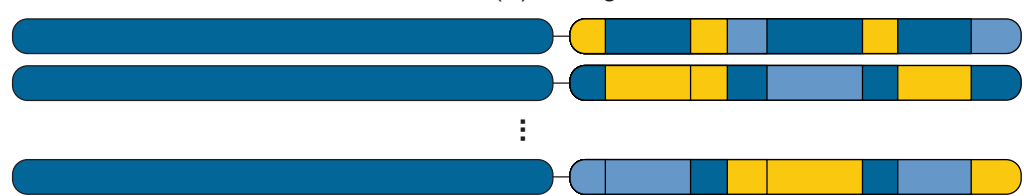

Fig. 1 Acyl-ACP reductase activity of natural and chimeric enzymes. a Alcohol-forming acyl-ACP reductases consist of two domains that sequentially reduce acyl-ACP substrates to aldehydes, and then aldehydes to alcohols. $\mathbf{b}$ We focused our studies on three diverse sequences from $M$. aquaeloei (dark blue), Marinobacter BSs20148 (light blue), and Methylibium Sp. T29 (yellow), which we refer to as A, B, and T, respectively. c Total fatty alcohol production by the three natural sequences and the six chimeric enzymes generated by shuffling their AHR and ATR domains. The error bars represent one standard deviation centered at the mean of four replicates $(n=4)$ from cultures derived from individual colonies, except for MA-ACR (where $n=5)$, parent B (fusion A-B, where $n=3$ ) and the empty vector $(n=2)$. d ATR domain residue-residue contact map used for SCHEMA recombination. The colored squares depict the eight sequence blocks from the SCHEMA design that minimize structural disruption. e The SCHEMA blocks mapped onto the ATR domain's three-dimensional structure (the colors correspond to the squares in (d)). f Our chimeric ATR library was fused to the AHR domain from MAACR. Source data underlying Fig. 1c are provided as a Source Data file. 
chimeric ATR domains with the highly active AHR domain from MA-ACR (Fig. 1f).

For the remainder of the paper, we refer to chimeras by a block sequence (e.g., A-ABTABTAB) that specifies which of the three enzymes each sequence fragment was inherited from. Here, $A, B$, and $\mathrm{T}$ correspond to MA-ACR, MB-ACR, and MT-ACR, respectively; the first position specifies the AHR domain and the remaining positions specify the ATR domain's eight SCHEMA blocks. We also refer to the three sequences that have all eight ATR blocks from a single natural enzyme as "parental" enzymes. Here "parent $A$ " has the block sequence AAAAAAAAA, "parent $\mathrm{B}$ " is $\mathrm{A}-\mathrm{BBBBBBBB}$, and "parent $\mathrm{T}$ " is A-TTTTTTTT.

Increasing fatty alcohol production with ML-driven enzyme engineering. We aimed to identify the most highly active enzymes from our chimeric ATR domain library. However, the chimera space consists of thousands of unique sequences and is much too large to fully characterize using our low-throughput gas chromatography assay. Instead, we developed an ML-based sequence optimization method to rapidly identify highly active sequences with minimal experimentation (Fig. 2a). Our approach consists of generating diverse initial sequence sampling to get a preliminary view of the landscape, followed by iterative design- test-learn cycles to efficiently search the landscape and discover optimized sequences.

We generated a diverse initial sampling of sequence space using a greedy algorithm to identify the set of 20 sequences that maximized the Gaussian mutual information with the full chimera space consisting of 4374 sequences. We then constructed these sequences and experimentally measured their fatty alcohol titers in three E. coli strains (Supplementary Fig. 2). We evaluated the chimeras' titers in RL08ara under aerobic conditions to assess activity on acyl-ACP substrates. Seventeen of the twenty sequences displayed no measurable alcohol production in RL08ara and the remaining three produced low titers that were below the least productive parent $(\mathrm{T})$. We also tested their activity in the CM24 strain $^{8}$ that was engineered to produce high concentrations of acyl-CoA substrates. In the CM24 strain under anaerobic conditions, we found two of the twenty chimeras produced alcohol titers comparable to least productive parent (B). Finally, we also evaluated alcohol titers in BL21(DE3) under aerobic conditions and found eight of the twenty chimeras produced measurable alcohols. Notably, the panel of twenty chimeras displayed differential activity across strains, which could be the result of varying substrate pools within each strain and different substrate preferences between the chimeric enzymes.

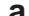

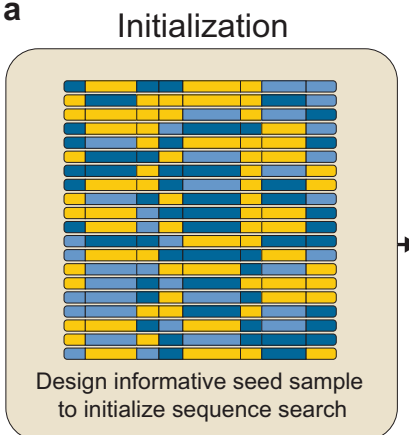

b
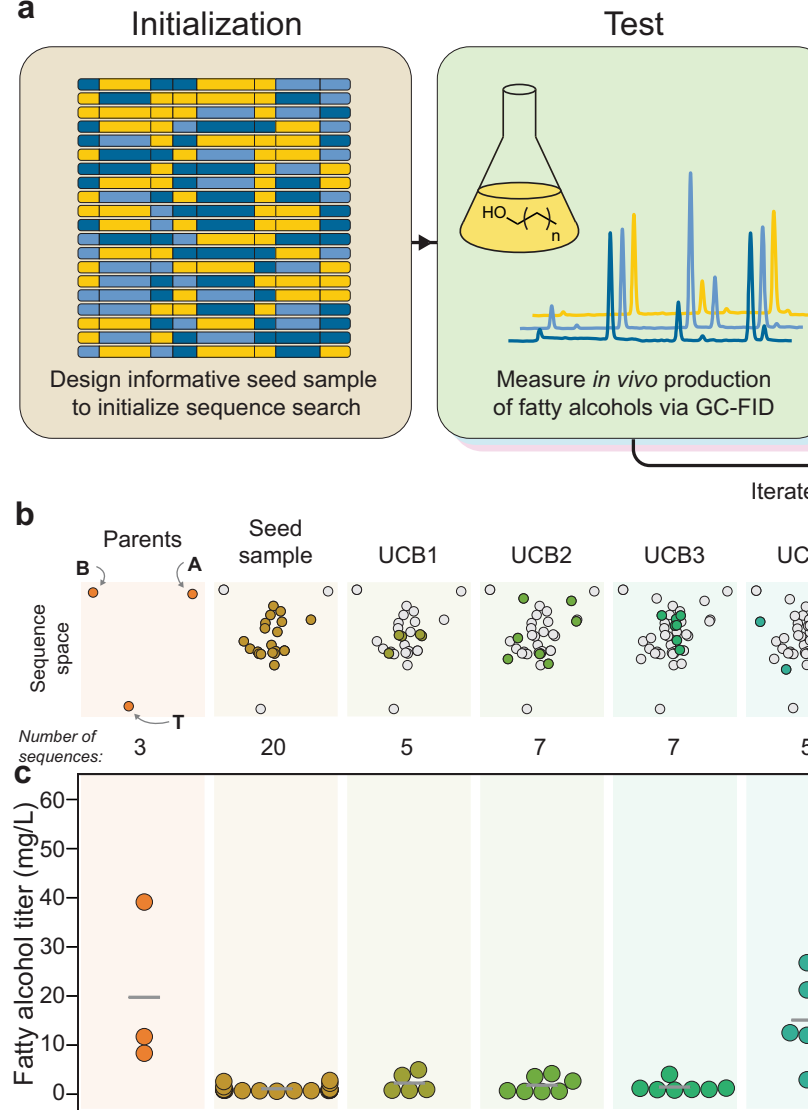

Measure in vivo production of fatty alcohols via GC-FID
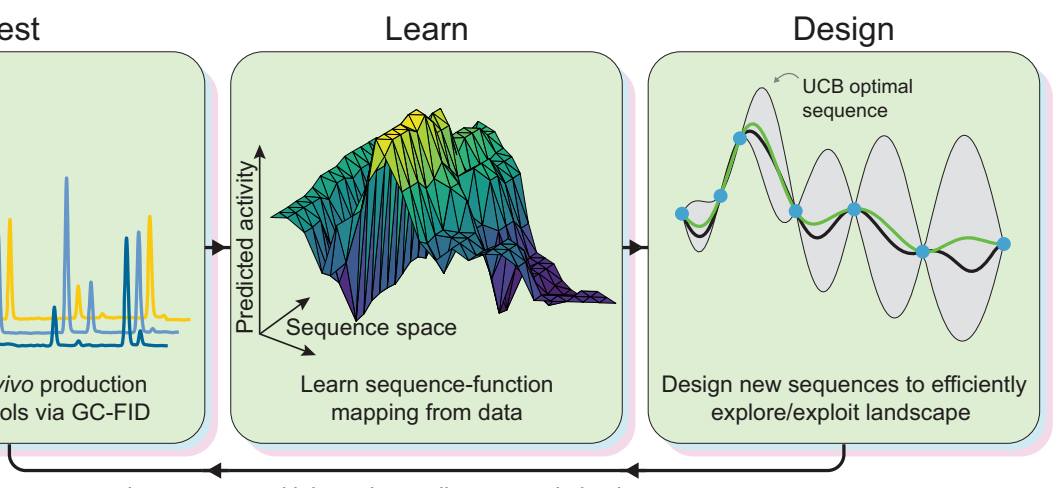

Iterate over multiple cycles to discover optimized enzymes
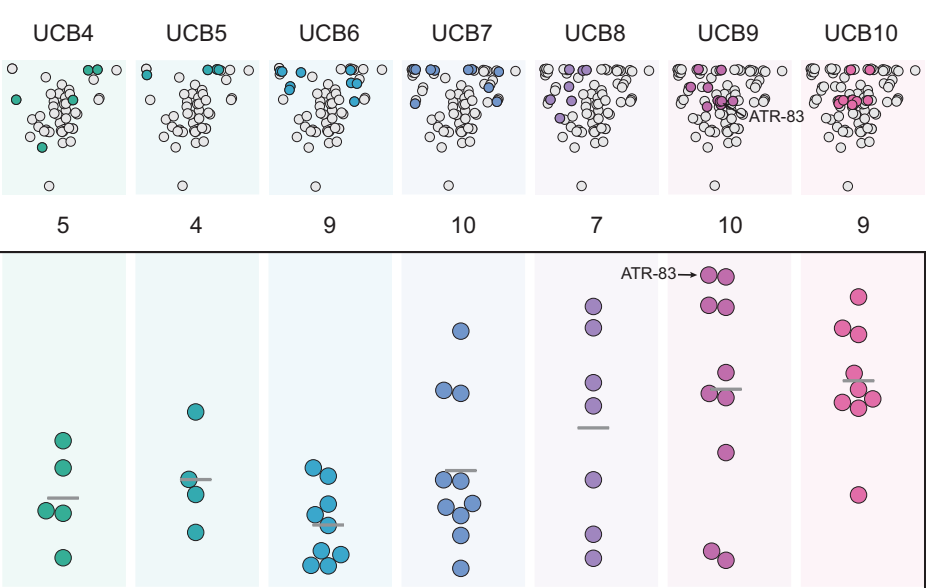

Fig. 2 ML-accelerated protein sequence optimization. a An overview of our sequence space search strategy. We first initialize the search by designing a diverse set of sequences that broadly sample the landscape. We then iterate through multiple design-test-learn cycles to efficiently understand and optimize in vivo fatty alcohol production. b Sequence space visualization over ten rounds of UCB optimization (each round is shown as a different color). The three parent enzymes are found at the vertices of this chimeric sequence space and all chimeras fall within the parents' envelope. The UCB optimization started by broadly sampling the landscape, but quickly converged on highly active regions. c The in vivo fatty alcohol titers over the course of the sequence optimization. Each point depicts an individual sequence's mean fatty alcohol production in the sequence optimization phase and the horizontal gray bars represent the average titer during that round of sequence optimization. The mean, standard deviation and number of replicates, where $n$ is equal to the number of cultures analyzed (each one from an individual colony), are shown in Supplementary Table 5. Source data underlying Fig. 2c are provided as a Source Data file. 
The fatty alcohol titer data from these 20 initial sequences was used to train Gaussian process (GP) sequence-function models that can make predictions across the entire chimera space. Importantly, GPs also provide estimates of the model's uncertainty (confidence intervals) that can be used to gauge the reliability of predictions and highlight gaps in its understanding of the landscape $\mathrm{e}^{14,15}$.

With the initialized GP sequence-function model, we then iterated through multiple design-test-learn cycles with the goal of identifying the optimal sequence with minimal experimental samples. The sequences for the next round of experimentation were designed using an upper-confidence bound (UCB) criterion that simultaneously explores uncertain regions of the landscape and samples sequences that are predicted to be optimized. UCB optimization provides strong theoretical guarantees for efficiently balancing exploration and exploitation ${ }^{25,26}$, and should rapidly converge on the optimal sequences. During each iteration, we designed 10-12 sequences using a batch mode UCB criterion (see "Methods"), assembled the corresponding genes, transformed them into E. coli, and measured each strain's fatty alcohol titer using gas chromatography. The new data was then used to update the sequence-function model and the process was repeated. We performed a total of ten rounds of UCB sequence optimization and saw gradual improvements in fatty alcohol titers (Fig. 2). The details of each round of UCB optimization can be found in Supplementary Table 4.

The UCB sequence optimization converged on multiple highly active acyl-ACP reductases. The enzyme with the highest titer had a block sequence of A-ATBBAAAB and we refer to this top sequence as ATR-83. Additional in vivo characterization showed that ATR-83 produces a total titer of $54 \pm 11 \mathrm{mg} / \mathrm{L}$ fatty alcohols (Supplementary Table 5), which is nearly fivefold greater than the titer of MA-ACR and about twofold greater than the best natural sequence (MB-ACR). The alcohols produced by ATR- 83 and the other top chimeras consisted of primarily hexadecanol (C16) and some tetradecanol (C14). This product distribution is expected since long chain acyl-ACPs are the primary precursors for the lipids that make up the cell membranes in E. coli ${ }^{27,28}$.

Improved fatty alcohol production occurs via an enhanced catalytic rate on acyl-ACP substrates. Our engineered acyl-ACP reductase chimeras produce several fold more fatty alcohols than the initial natural sequences. Increased flux through the metabolic pathway can be the result of improved protein stability and/or expression, enzyme kinetic properties, or possibly interactions with other components of the pathway. We performed further biochemical analysis of the engineered enzymes to better understand how they increase alcohol production.

We first measured the level of enzyme expression in the production strain (Supplementary Fig. 3, Fig. 3a). We found all sequences were expressed at high levels and there were no statistically significant differences between the natural and engineered sequences. Next, we purified the enzymes and measured their kinetic properties on palmitoyl-ACP (Fig. 3b, c). ATR-83 and parent B displayed similar $K_{M}$ values for palmitoylACP, but ATR-83 had a substantially larger turnover number. ATR-83's increase in $k_{\text {cat }}$ matches its improvements in fatty alcohol titer. Taken together with the enzyme expression data, this suggests that the engineered enzymes are increasing alcohol production by an enhanced catalytic rate.

We also analyzed the enzymes' activity on CoA substrates and found that ATR-83 has a lower activity than the parents on palmitoyl-CoA (Supplementary Fig. 4). This suggests that ATR83 may not be a faster enzyme overall, but instead displays an altered preference for ACP over CoA. This altered preference could be the result of changes in the protein surface that interacts with the ACP substrate.

Statistical analysis of the enzyme landscape reveals features that influence fatty alcohol production. Over the course of our UCB sequence optimization, we collected 96 data points mapping chimeric sequences to fatty alcohol titers. This sequence-function data can serve as a rich resource for understanding how protein sequence and structure impact in vivo enzyme activity. We trained a GP regression model to predict fatty alcohol titers from sequence. This model displayed excellent predictive ability in a cross-validation test (Supplementary Fig. 5).

We used this predictive model to assess how each chimera sequence block contributes to overall enzyme activity (Fig. 4a). We see that most block positions influence activity and display a broad range of effects. The three sequence blocks with the largest positive contribution were block 7 from MA-ACR, block 3 from MB-ACR, and block 2 from MT-ACR. Substitution to any one of these blocks tends to increase alcohol titers by over $70 \%$. Block 8 from MB-ACR also strongly tends to increase the titers. The sequence blocks with the most negative contribution were blocks 3 and 7 from MT-ACR. Overall, most blocks from MT-ACR were deleterious for alcohol production.

We mapped the block effects onto MA-ACR's homology model to relate their contributions to structure and mechanism (Fig. 4b). Block 2 likely forms extensive interactions with the enzyme's NADPH cofactor, and MT-ACR is the best parent at this position. While there are many amino acid differences in this block, it's notable that MT-ACR has a different NADPH binding motif than the other two parents (GGSSGIG vs GATSGIG). MTACR's motif may provide more efficient NADPH utilization in vivo. Blocks 4-8 make up the binding pocket for the acylthioesters. Block 5 contains three of the catalytic residues (a Y, S and $\mathrm{K}$ ), and block 6 , whose sequence is highly conserved, appears to be involved in NADPH binding. Blocks 7 and 8 appear to contain surface residues; positively charged residues in these blocks are likely involved in docking the negatively charged acyl$\mathrm{ACP}^{29}$

We hypothesized the net charge of the enzymes' substrate binding pocket may influence activity because the ACP substrate contains many negatively charged residues. To examine the enzymes' charge distribution near the substrate binding site, we computationally docked ACP (from PDB entry 6DFL) to our homology model of MA-ACR using RosettaDock ${ }^{30}$. We then identified all interface positions within a $10 \AA$ radius of the docked ACP and calculated the net charge of each chimera's interface residues. We found the net charge of an enzyme's substrate binding interface was positively correlated with the total fatty alcohol titer (Fig. 4c). A chimera's substrate interface charge is dictated by nine sequence positions that are near the ACP substrate and that contain charged residues in at least one parent (Fig. $4 \mathrm{~d}, \mathrm{e}$ ). The charges at these sequence positions can largely explain the preferred blocks from Fig. 4 a.

\section{Discussion}

Engineering fatty acyl reductases (FARs) to have improved activity on acyl-ACP substrates could open routes to in vivo production of fatty alcohols, and other valuable bioproducts such as waxes and alkanes. In this work, we engineered enzymes with improved activity on acyl-ACP substrates. Our approach leveraged gene shuffling to broadly sample sequence space and MLdriven protein engineering to rapidly and efficiently identify optimized sequences. Our top identified enzyme, ATR-83, displayed twofold higher in vivo fatty alcohol titer than the best natural sequence, MB-ACR, and nearly fivefold higher titers than 


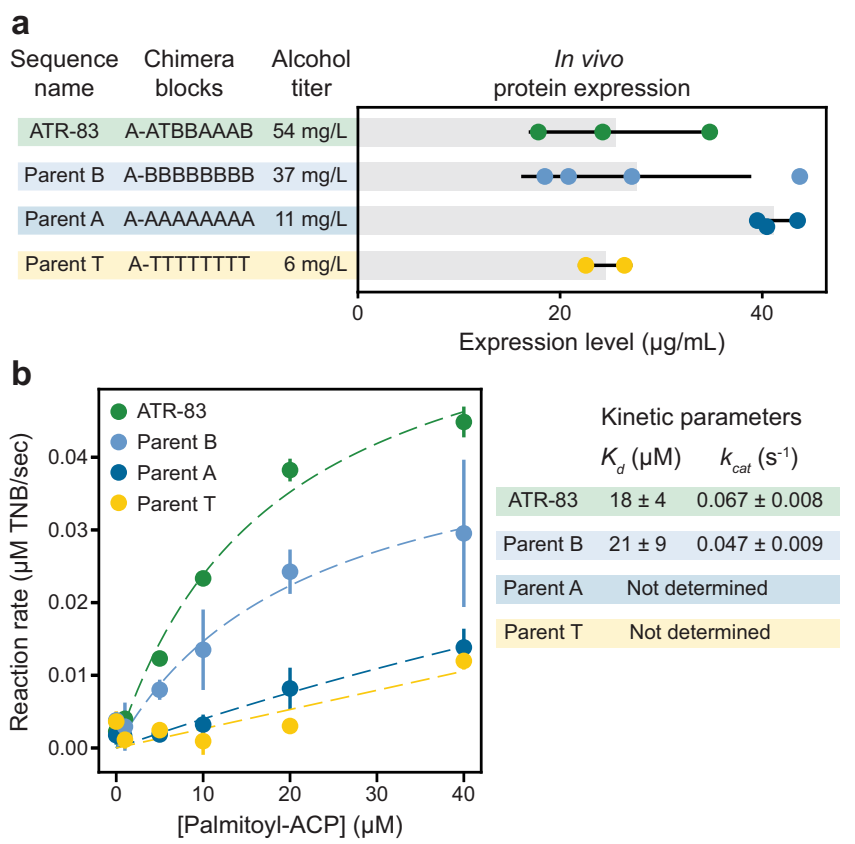

Fig. 3 Expression levels and kinetic activity of selected ATRs. a We measured the expression levels of the ATR-83 chimera and the three parental enzymes. These four enzymes displayed no significant differences in expression despite the large differences in their alcohol titers. The error bars represent one standard deviation centered at the mean $(n=3,4,3$, and 2 for ATR-83, parent B, parent A and parent T, respectively, where $n$ is the number of cultures analyzed, each from individual colonies). b We characterized the kinetics of selected enzymes on palmitoyl-ACP. The error bars represent one standard deviation centered at the mean ( $n=4$ technical replicates). ATR-83 displayed a higher turnover number $\left(k_{\text {cat }}\right)$ relative to parent B, and higher activity overall compared to the other parents. The kinetic parameters for parents $A$ and $T$ could not be precisely determined due to their low overall activity and the resulting poor fit to the Michaelis-Menten model. Source data are provided as a Source Data file.

MA-ACR. These increases in fatty alcohol titer are a result of ATR-83's enhanced turnover number on ACP substrates. The chimeric enzymes discovered in this work have potential to improve the efficiency of alcohol production from acyl-ACPs in vivo.

Shuffling the AHR and ATR domains between the three natural sequences generated chimeric enzymes that produce a broad range of fatty alcohol titers. From these results, it appears the ATR domain from MB-ACR has the highest activity on ACP substrates and the AHR domain from MA-ACR has the highest activity on the intermediate aldehyde substrate. Rather than directly affecting the catalytic rate, it's also possible that these domains could be enhancing activity through inter-domain interactions, especially since MA-ACR has been shown to be tetrameric ${ }^{4}$.

Machine learning is rapidly advancing the fields of directed evolution and protein engineering ${ }^{15-17,31}$. Though some MLbased strategies (especially those involving deep learning or neural nets) require massive amounts of training data, activelearning approaches (such as UCB optimization) can be used to simultaneously explore the sequence-function landscape and identify improved sequences from relatively few data points. The reduced need for data enables protein engineering workflows that do not depend on high-throughput techniques, and thus overcomes major limitations of directed evolution approaches. Our design-test-learn cycle closely resembles the UCB optimization process previously used to engineer thermostable chimeric cytochrome P450s ${ }^{14}$. However, a key difference in this work was the introduction of an active/inactive binary classifier to filter out potential inactive sequences that provide little information regarding enzyme activity. Incorporating this classifier led to improved predictions by the GP regression model, especially in early UCB rounds when the number of active sequences was small (only 12 sequences were active from the first three rounds).
In the early rounds of our UCB sequence optimization, we found it was helpful to restrict the number of block exchanges from the parent sequences in order to bias the search towards functional sequences. Sampling further away almost always resulted in non-functional sequences that provided little information about the fatty alcohol production landscape. We learned this trick during the course of the sequence optimization, which certainly limited the efficiency of our method. Future improvements to UCB algorithm could include an informative prior for the active/inactive binary classifier that encodes a preference to sample near the parent sequences when limited functional data is available.

In principle, our protein engineering framework is applicable whenever an underlying fitness landscape can be inferred via machine learning. There have been multiple previous studies demonstrating the effectiveness of machine learning to navigate the sequence-function landscape. A notable example used a similar UCB method to optimize cytochrome P450 thermostability ${ }^{14}$. A lower confidence bound (LCB) algorithm was used to predict chimeric channelrhodopsin sequences that localize to the plasma membrane of mammalian cells, and UCB optimization was then used to identify chimeras with high localization ${ }^{18}$. GP classification and regression models were further used to engineer highly light sensitive channelrhodopsins for optogenetics ${ }^{31}$. Iterative searches through protein sequence-function landscapes such as UCB optimization and LCB minimization reduce dependency on large datasets, and enable engineering of more difficult protein targets.

ATR-83 produced $50 \%$ more fatty alcohols than parent $\mathrm{B}$ (ABBBBBBBB) and 4.9-fold more than MA-ACR. It is difficult to interpret these in vivo results because intracellular acyl-ACP pools exist as a broad mixture from $\mathrm{C} 4-\mathrm{C} 18$, and each enzyme may have its own substrate preferences. We performed further kinetic characterization on the enzymes and found ATR-83's increased in vivo alcohol production is the result of enhanced 
a

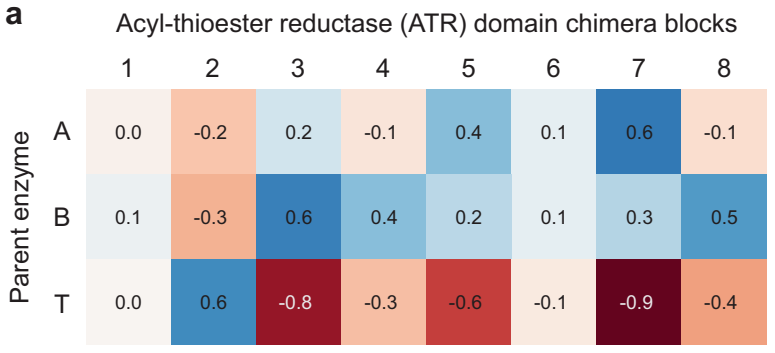

C

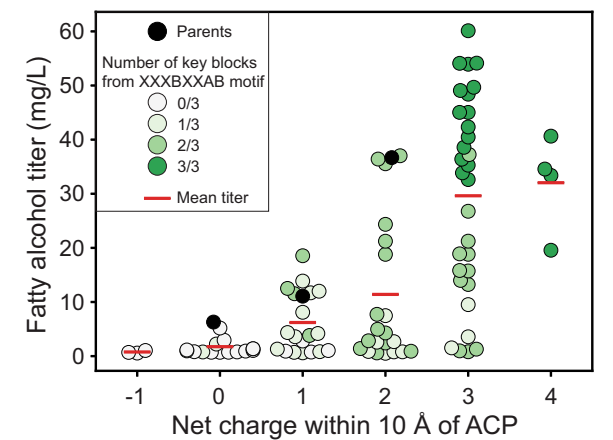

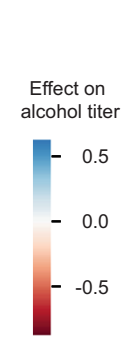

d

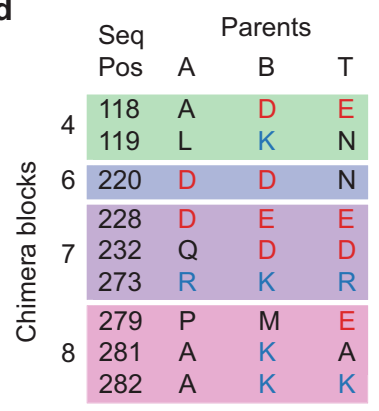

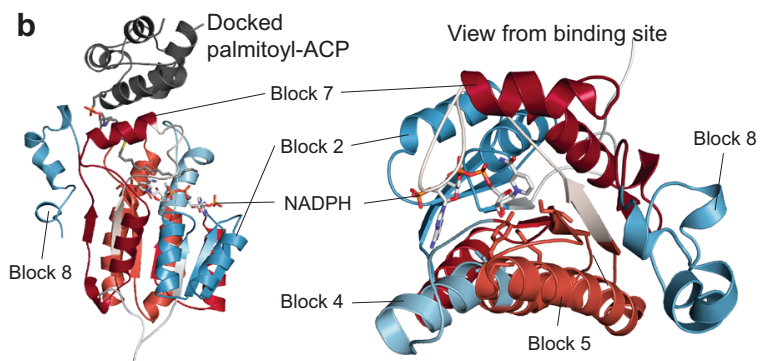

e

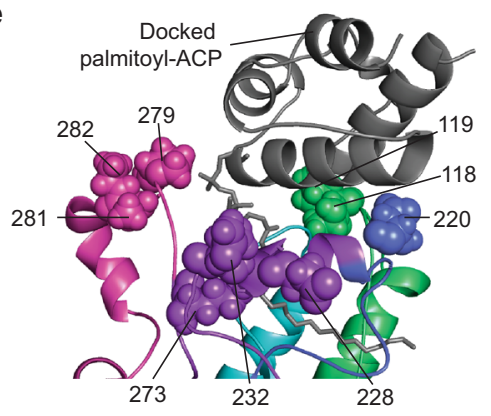

Fig. 4 Statistical analysis of the fatty alcohol production landscape. a Contributions of each sequence block to the alcohol production of chimeras. b Mapping of the contributions to the structural model of MA-ACR. Blocks with strong effects (either positive or negative) line up with key structural features such as the NADPH binding site, the active site and the ACP binding site. c Correlation between net charge near the binding site and fatty alcohol titer of chimeras. Chimeras with higher net charges tend to produce more alcohols. In addition, three key blocks were found to correlate with both activity and with charge. Combining all three of these blocks results in highly active enzymes. The statistics for the finalized dataset (mean, standard deviation and number of replicates) are available in Supplementary Table 5. $\mathbf{d}$ Positions in the parent sequence alignment that contain non-conserved charged residues within $10 \AA$ of the putative ACP binding site (positively charged residues are shown as blue text, negatively charged residues are shown as red text). e Locations of key charged residues in the structural model of MA-ACR docked with palmitoyl-ACP. Optimal combinations of blocks could produce more favorable interactions with the ACP. Source data underlying Fig. 4c are provided as a Source Data file.

turnover number $\left(k_{c a t}\right)$ on ACP substrates, rather than enzyme expression or $K_{M}$ effects. Interestingly, ATR-83 displays lower activity on acyl-CoA substrates than parent B and MA-ACR. Since both acyl-ACP and acyl-CoA substrates have the same thioester bond that is being reduced, one might expect substrate specificity to manifest as differences in $K_{m}$ between the enzymes. However, we observed enzymes' $k_{\text {cat }}$ to be the major determinant of substrate specificity. One possible explanation for the observed behavior could be that ACP is interacting with the enzyme surface to allosterically enhance the catalytic rate. Similar allosteric modulation by ACPs has been observed in the LovD enzyme ${ }^{32}$.

We found a positive correlation between an enzyme's net charge near the putative substrate binding site and its activity on acyl-ACPs in vivo. This relationship may be expected because positive charges on the enzyme surface could enhance electrostatic interactions with the negatively charged ACP substrate. The chimeric enzymes' substrate interface charges are largely dictated by blocks 4,7 , and 8 . Sequences with $\mathrm{B}$ at block 4 , A at block 7 , and $\mathrm{B}$ at block 8 (i.e., XXXBXXAB) can increase the net interface charge of a chimera by up to +4 . The average alcohol titer of chimeras containing these three blocks is $42 \mathrm{mg} / \mathrm{L}$, compared to an average of $8 \mathrm{mg} / \mathrm{L}$ for sequences without that combination. These results suggest future enzyme engineering directions to supercharge the substrate interface with positively charged residues to further enhance electrostatic interactions with ACP. A similar approach has been applied to acyl-ACP thioesterases, leading to improved enzyme activity ${ }^{29}$.

While we demonstrated that our engineered enzymes have improved activity on palmitoyl-ACP both in vivo and in vitro, the activity of the enzymes on shorter and medium chain substrates is less clear. Production of medium chain fatty alcohols, such as octanol, remains a prime target for metabolic engineering, since medium chain fatty alcohols are more valuable than long chain fatty alcohols ${ }^{33}$. In order to explore the in vivo activity of these engineered enzymes on shorter chain acyl-ACPs, new methods would be needed to alter the acyl-ACP distribution in the cells without significantly disrupting pathways involving production of lipids for the cell membranes. Alternatively, pathways that utilize acyl-CoA pools show promise for making medium length alcohols selectively ${ }^{7,9}$. While our active-learning strategy focused on acyl-ACP activity, it could also be used to enhance activity on medium chain acyl-CoAs.

While our engineered ATRs were able to significantly boost fatty alcohol production from acyl-ACP substrates, the titers we achieved are still far below those from pathways that rely on acylCoA intermediates, such as the implementation of reverse beta oxidation in Mehrer et al. $(1.8 \mathrm{~g} / \mathrm{L})^{8}$ and the utilization of a thioesterase/acyl-coA ligase pair in Hernández-Lozada et al. $(1.3 \mathrm{~g} / \mathrm{L})^{9}$. Our lower titers are expected since the acyl-ACP pool is considerably smaller than the acyl-CoA pools that can be achieved in these and similar pathways. In addition, these previous works involved extensive strain optimization to boost acylCoA pools, while our current enzyme engineering results were achieved in an unmodified host strain. Importantly, the acyl-ACP route to produce fatty alcohols is more direct and has a lower energetic cost than pathways utilizing acyl-CoA intermediates. Future work could focus on strain engineering efforts to upregulate fatty acid biosynthesis by modifying FadR expression or by relieving the pathway's feedback inhibition by longer chain acylACPs.

Our ability to engineer microbes to produce high-value chemicals is often limited by the availability of enzymes to catalyze key chemical reactions. We have presented an enzyme engineering framework that leverages ML-based sequence-function 
models with iterative experimentation to rapidly identify improved enzymes. This approach can be generally applied to enzymes that lack a high-throughput functional assay or structural information, and therefore are challenging to engineer using traditional directed evolution and rational methods. Future advances in enzyme engineering will open routes to produce valuable chemicals from low-cost and renewable feedstocks.

\section{Methods}

Chemicals, reagents, and media. E. coli RL08ara ${ }^{21}$ and CM24 ${ }^{8}$ assay media used for this study are the same composition as Miller LB, except with $10 \mathrm{~g} / \mathrm{L}$ peptone instead of $10 \mathrm{~g} / \mathrm{L}$ tryptone. CM24 media was supplemented with $1 \% \mathrm{w} / \mathrm{v}$ glucose, and sterile filtered using a $2 \mu \mathrm{M}$ filter. E. coli RL08ara assay medium was sterilized by autoclaving. Both media were adjusted to a $\mathrm{pH}$ of 7.0 prior to sterilization.

Individual fatty alcohol standards were prepared at a concentration of $100 \mathrm{mg} / \mathrm{mL}$ by dissolving alcohols ranging from $\mathrm{C} 3$ to $\mathrm{C} 17$ in 200 proof ethanol. Then, alcohols were mixed to make $10 \mathrm{mg} / \mathrm{mL}$ standards of even-chain alcohols (C4, C6, C8, C10, C12, $\mathrm{C} 14$, and C16) and odd-chain alcohols (C3, C5, C7, C9, C11, C13, C15, C17). All unique biological materials are available upon request.

Measuring in vivo fatty alcohol titers. We measured in vivo alcohol titers produced by each enzyme variant using gas chromatography (GC). Overnight cultures started in LB + Kanamycin from individual colonies from the transformation were grown for $16-20 \mathrm{~h}$ and diluted into a $50 \mathrm{~mL}$ culture of E. coli RL08ara Assay Medium + Kanamycin in a $250 \mathrm{~mL}$ baffled shake flask such that the final OD was about 0.01 . The media had a $20 \%(10 \mathrm{~mL})$ dodecane overlay, and we supplemented the media with $1 \mathrm{~mL}$ of $50 \% \mathrm{v} / \mathrm{v}$ glycerol. The cultures grew at $37^{\circ} \mathrm{C}$ for $45 \mathrm{~min}$ at $250 \mathrm{rpm}$, and then we induced protein expression by adding $500 \mu \mathrm{L}$ of $100 \mathrm{mM}$ IPTG (final concentration $100 \mu \mathrm{M}$ IPTG). As a control, each batch also included blank cultures that were prepared by mixing media, dodecane, glycerol and antibiotic in the same amounts as the expression cultures, but without any cells added. The expression cultures incubated for $18 \mathrm{~h}$ at $30{ }^{\circ} \mathrm{C}$ after induction.

Afterwards, we cooled the expression cultures on ice to prevent evaporation. Then, we added $150 \mu \mathrm{L}$ of $10 \mathrm{mg} / \mathrm{mL}$ odd-chain internal standard mixture to each culture flask and mixed them vigorously to make an emulsion. Immediately after mixing, we transferred $5 \mathrm{~mL}$ of the emulsion to a glass centrifuge tube pre-loaded with $1 \mathrm{~mL}$ of n-hexanes. We vortexed the tubes for $20 \mathrm{~s}$, shook for $20 \mathrm{~s}$, and vortexed for another $20 \mathrm{~s}$. Then, we centrifuged the samples for about $10 \mathrm{~min}$ until the organic layer and aqueous layers separated and extracted about $900 \mu \mathrm{L}$ of the organic layer to load into a GC vial for analysis on GC-FID.

We analyzed all GC samples using a Shimadzu Model 2010 GC-FID system with an AOC-20i autosampler and a $60 \mathrm{~m} 0.53 \mathrm{~mm}$ ID Stabilwax column (Restek 10658). The oven temperature program used to analyze samples from RL08ara and CM24 samples was based on Mehrer et al. ${ }^{8}$ and is as follows: $45^{\circ} \mathrm{C}$ hold for $10 \mathrm{~min}$, ramp to $250{ }^{\circ} \mathrm{C}$ at $12{ }^{\circ} \mathrm{C} /$ minute, hold at $250{ }^{\circ} \mathrm{C}$ for $10 \mathrm{~min}$. In some individual experiments we shortened the hold time. Each run included standards of the oddchain internal standard mixture and even-chain standard mixture to control for any changes in the retention times of the analytes. We estimated the concentrations of even-chain fatty alcohols by averaging the areas $\left(A_{i-1}\right.$ and $\left.A_{i+1}\right)$ and concentrations $\left(\mathrm{C}_{\mathrm{i}-1}\right.$ and $\left.\mathrm{C}_{\mathrm{i}+1}\right)$ of the odd-chain internal standards that bracketed the particular even-chain analyte. We used the resulting response factor to convert the area of the even-chain species $\left(\mathrm{A}_{\mathrm{i}}\right)$ to the original media concentration $\left(\mathrm{C}_{\mathrm{i}}\right)$ per the following equation:

$$
C_{i}=A_{i} * \frac{\operatorname{avg}\left(\mathrm{C}_{\mathrm{i}-1}, \mathrm{C}_{\mathrm{i}+1}\right)}{\operatorname{avg}\left(\mathrm{A}_{\mathrm{i}-1}, \mathrm{~A}_{\mathrm{i}+1}\right)}(i=2,4,6,8,10,12,14,16)
$$

Aerobic alcohol production in BL21(DE3). We cloned the initial seed sample ACR chimeras into the pET28 backbone and transformed into BL21(DE3). Cultures were started in $\mathrm{LB}+$ Kanamycin from individual colonies from the transformation and grown overnight for 16-20 h. We diluted the cultures 100 -fold into $5 \mathrm{~mL}$ cultures of $\mathrm{LB}+$ Kanamycin in culture tubes. We grew the cultures for $2.5-3 \mathrm{~h}$, measured the ODs, and then induced with $5 \mu \mathrm{L}$ of $100 \mathrm{mM}$ IPTG and incubated for $24 \mathrm{~h}$ at $20^{\circ} \mathrm{C}$ with shaking at $250 \mathrm{rpm}$.

Following protein expression, we incubated the cultures on ice for $1.5-2.5 \mathrm{~h}$. Nonanol (C9) and heptadecanol (C17) were used as internal standards; a solution that was $5 \mu \mathrm{M}$ nonanol and $5 \mu \mathrm{M}$ heptadecanol in hexanes was prepared and added $(1 \mathrm{~mL})$ to each $5 \mathrm{~mL}$ expression culture. We then vortexed and spun down the sample in a centrifuge (1000x G for $10 \mathrm{~min}$ ) to separate the phases. In total, $900 \mu \mathrm{L}$ of the organic layer was extracted for analysis on GC-FID. Titers of fatty alcohols were determined using an external standard curve with standards of each of the even chain fatty alcohols in hexanes and dividing by the extraction ratio (5) to convert from the concentration in the organic phase to the original concentration in the media.

Anaerobic alcohol production in CM24. ACR chimeras were cloned into the pBTRCK plasmid backbone and transformed into CM24 along with seFadBA (g130, pACYC-seFadBA) and tdTER (g131, pTRC99A-tdTER-fdh) ${ }^{8}$. We started overnight cultures from individual colonies in LB + Kanamycin + Carbenicillin + Chloramphenicol. The following day, after $16-20 \mathrm{~h}, 600 \mu \mathrm{L}$ of overnight cultures were diluted in $30 \mathrm{~mL}$ of CM24 Assay Medium + Kanamycin + Carbenicillin + Chloramphenicol with a $20 \%(6 \mathrm{~mL})$ dodecane overlay in a $50 \mathrm{~mL}$ serum vial, which was sealed. We grew the cultures for $2 \mathrm{~h}$ at $30^{\circ} \mathrm{C}$, and then induced by injecting $300 \mu \mathrm{L}$ of $100 \mathrm{mM}$ IPTG (for a final IPTG concentration of $\sim 100 \mu \mathrm{M}$ ) through the septum with a needle. Cultures were then incubated at $30^{\circ} \mathrm{C}$ for $48 \mathrm{~h}$

Following expression, we cooled the cultures on ice and added $180 \mu \mathrm{L}$ of an internal standard mixture (the same fatty alcohol mixture used for quantitation of alcohols in RL08ara). We mixed the samples thoroughly and extracted $5 \mathrm{~mL}$ of the emulsion with $1 \mathrm{~mL}$ of hexane per the same protocol as RL08ara above.

Structural modeling and SCHEMA library design. We utilized the MODELLER ${ }^{34}$ homology modeling software to build 100 models of each of the acyl-thioester reductase domains of MA-ACR, MB-ACR, and MT-ACR using the following PDB entries as templates: 3M1A-A, 3RKR-A, 3RIH-A, 3AFM-B, 3AFN-B, and 4BMV-A. We built a contact map by determining which pairwise amino acid contacts (defined as two amino acids within a $4.5 \AA$ radius based on any atoms in the amino acids) were present in each model, and weighted each contact by the percentage of models in which the contact was present.

We determined the crossover between the aldehyde-reductase domain and the acyl-thioester reductase (ATR) domain by aligning the sequences of MA-ACR, MB-ACR, and MT-ACR and selecting a crossover point at the conserved LDPDL, $\sim 350-360$ residues from the N-termini. Then, we used SCHEMA-RASPP to determine 7 additional crossover locations within the ATR domain that were compatible with Golden Gate assembly.

Gene assembly and strain construction. All ATR enzymes tested were cloned into the pBTRCK plasmid backbone and transformed into E. coli RL08ara ${ }^{21}$. We obtained the three natural parent sequences from prior studies ${ }^{8,9}$. We amplified the AHR and ATR domains of each of the natural sequences, as well as the plasmid backbone, by PCR using primers (Supplementary Table 7) that contained Golden Gate overhangs. We used Phusion Hot Start Flex 2X Master-Mix (NEB) for all PCR reactions. Then, we used Golden Gate assembly to combine the pieces and synthesize the domain shuffled variants. Golden Gate reactions were carried out either using commercial Golden Gate assembly mix (NEB), or an in-house mixture of the components from NEB (T4 DNA ligase buffer, BsaI HF v2 and T4 DNA ligase).

We designed plasmids containing each of the 24 blocks determined by RASPP such that each block was flanked by BsaI restriction sites. The plasmids were synthesized by TWIST Biosciences. The blocks (including the BsaI site) were amplified by PCR and cloned into a backbone vector harboring the AHR domain of MA-ACR by Golden Gate assembly. For sequences that we studied in vitro, we amplified the whole FAR sequence and used Golden Gate assembly to add the insert into a pET 28 backbone.

Greedy algorithm to design an informative seed sample. We sought to identify the set of 20 chimera sequences that is maximally informative of the full chimera landscape. We quantify "informativeness" as the Gaussian mutual information $I(S ; L)$ between the chosen sequences $S$ and the full landscape $L$. This mutual information simplifies to the Gaussian entropy $H(S)$ because $S$ is a subset of $L$. Entropy is a submodular set function and can therefore be efficiently optimized using a greedy algorithm.

We started with our three parent sequences and scanned over all possible chimera sequences $s_{i}$ to determine which resulted in the largest Gaussian entropy $H\left(S \cup\left\{s_{i}\right\}\right)$. This top sequence was added to the chosen set of sequences $S$ and the greedy sequence selection process was repeated until 20 sequences were chosen.

Sequence-function machine learning. We modeled the sequence-function landscape using a combination of a Gaussian Naive Bayes (GNB) classifier to distinguish inactive versus active sequences and Gaussian process (GP) regression to model a sequence's fatty alcohol titer

The active/inactive classifier was trained on chimera sequence-function data using scikit-learn's Naïve Bayes classifier. We categorized sequences as active if their alcohol titer was above a certain threshold; otherwise, they were considered inactive. The amino acid sequences for each tested chimera were one-hot encoded and used as inputs for the classifier. The resulting model provides a prediction of the probability that a sequence is an active enzyme.

We also trained a GP regression model on the active sequences' fatty alcohol titers. Our GP regression model used a homogeneous linear kernel to define the covariance between pairs of sequences

$$
k_{i, j}=\sigma^{2} \mathbf{x}_{i} \cdot \mathbf{x}_{j}
$$

where $\sigma^{2}$ is a tunable variance hyperparameter, and $\mathbf{x}_{i}$ and $\mathbf{x}_{j}$ are the encodings for sequences $i$ and $j$, respectively. The Hamming kernel one-hot encoded each amino acid option at each sequence position, while our structure kernel one-hot encoded amino acid combinations at each residue-residue pair that was contacting in the three-dimensional structure. We calculated the GP's posterior mean and variance following Algorithm 2.1 in Rasmussen \& Williams ${ }^{35}$ (Supplementary Method 1). 
We used leave-one-out cross-validation to scan variance $\left(\sigma^{2}\right)$ hyperparameter values ranging from $10^{-6}$ to $10^{5}$ and selected values that maximized the correlation coefficient and minimized the mean squared error (Supplementary Fig. 6). When these two objectives could not be realized simultaneously, we chose $\sigma^{2}$ values that balanced them. We then used the chosen $\sigma^{2}$ value to fit the GP model on all the data and predict the activities of all untested sequences that the GNB classifier labeled as active.

Upper-confidence bound optimization. We utilized UCB optimization to select informative sequences to build and test for the next round. For UCB rounds 2-10, we trained the active/inactive GNB classifier and the alcohol titer GP regression model on all prior data. We then applied the GNB and GP models to make functional predictions over all untested chimeras. We chose a panel of sequences to test using a "batch mode" UCB selection strategy ${ }^{36}$, while excluding any sequences that were predicted to be inactive from the GNB classifier. We first chose the sequence that maximized the GP upper confidence bound (mean + one standard deviation). This is the UCB optimal sequence. We then retrained the GP model with the assumption that the UCB optimal sequence's true titer was equal to its predicted titer. We then recalculated the UCBs and chose the new UCB optimal sequence. This process enables selection of multiple UCB optimal sequences per round, and it was repeated until 10-12 sequences were chosen per batch. The details of each round of UCB optimization can be found in Supplementary Table 4.

The first UCB round was performed slightly differently than the others because we were still refining our method. For the first UCB round, we trained GP regression models on alcohol titers from both BL21(DE3) and CM24 strains. We chose sequences that maximized the sum of the BL21(DE3) and CM24 UCB scores and selected a panel of ten chimeras using the batch mode UCB approach described above.

Measuring in vivo enzyme expression levels using SDS-PAGE. To verify that increases in fatty alcohol titers were due to enzyme activity, we performed additional characterization of the protein expression levels for the parents and selected chimeras. To estimate the expression level of the ATR enzyme, we performed additional replicates using the same expression conditions as were used during UCB optimization. Then, after extracting the fatty alcohols, we suspended the remaining $5 \mathrm{~mL}$ pellet in $1 \mathrm{~mL}$ of media. We normalized the ODs of the suspensions to an OD of 10 and pelleted and froze $500 \mu \mathrm{L}$ of the OD 10 culture. We later thawed the frozen pellets and lysed them using $250 \mu \mathrm{L}$ lysis buffer $(3872 \mu \mathrm{L}$ $100 \mathrm{mM}$ Tris $\mathrm{pH}$ 7.4, $120 \mu \mathrm{L}$ Bugbuster, $4 \mu \mathrm{L}$ lysozyme and $4 \mu \mathrm{L}$ DNAse I).

We prepared a standard curve using dilutions of purified MA-ACR. We added $3 \mu \mathrm{L}$ of each MA-ACR dilution to $12 \mu \mathrm{L}$ of SDS master mix (which consisted of 5 parts $2 \mathrm{X}$ SDS mix and 1 part $1 \mathrm{M}$ DTT) and mixed them in a 1:1 ratio (volume:volume) with empty vector lysate. The other lysates were mixed with $2 \mathrm{X}$ SDS buffer and $3 \mu \mathrm{L} 100 \mathrm{mM}$ Tris $\mathrm{pH} 7.4$ (to ensure equal volumes of lysate between the standards and the samples). We heat denatured the lysates (at $85^{\circ} \mathrm{C}$ for $2-5 \mathrm{~min}$ ) and analyzed them by SDS-PAGE.

We used FIJI, an image analysis software ${ }^{37}$, to estimate the intensities of the ATR band in the MA-ACR standards and generate a standard curve (Supplementary Fig. 3). We made new standard curves for each replicate to reduce gel to gel variability, and only compared samples to standards on the same page gel. Expression levels are reported as $\mu \mathrm{g} / \mathrm{mL}$ of ATR (at an OD of 20)

Biosynthesis of fatty acyl-ACP substrates. We synthesized the acyl-ACP substrates by functionalizing purified E. coli ACP with a 4'-phosphopantetheine arm by the acyl-ACP synthetase from Vibrio harvey ${ }^{38}$, and then attaching the acylchain to the thiol end of the arm using a phosphopantetheinyl transferase (SfP) from Bacillus subtilis.

Expression of V. harveyi AasS, B. subtilis SfP and E. coli ACP. The enzymes needed to functionalize palmitoyl-ACP were expressed using the method in Hernández-Lozada et al. with some minor modifications ${ }^{39}$. The cells were grown for $2 \mathrm{~h}$ at $37^{\circ} \mathrm{C}(200 \mathrm{rpm})$ and then induced with $1 \mathrm{mM}$ IPTG (final concentration) without cooling the cultures as was done in Hernández-Lozada et al. AasS and SfP were expressed overnight at $18{ }^{\circ} \mathrm{C}$ for $18-24 \mathrm{~h}$, and ACP was expressed at $20^{\circ} \mathrm{C}$ overnight $(18-24 \mathrm{~h})$ and harvested by centrifugation. We also purified the proteins using the method from Hernández-Lozada et al., however rather than using dialysis, we used Amicon filter columns to carry out buffer exchange. The final concentrations of the proteins were determined using Bradford assays.

Functionalization of E. coli ACP. To cleave the His-tag from the apo-ACP, we added $700 \mathrm{uL}$ of $2.1 \mathrm{mg} / \mathrm{mL}$ TEV protease to the $4 \mathrm{~mL}$ ACP solution. The reaction incubated overnight $(16-20 \mathrm{~h})$ at $20^{\circ} \mathrm{C}$ shaking at a speed of $250 \mathrm{rpm}$. At the conclusion of the digestion, we stored the mixture in $50 \%$ glycerol at $-80^{\circ} \mathrm{C}$. Later, to purify the cleaved apo-ACP, we thawed the digestion and ran it over parallel gravity columns packed with Nickel Sepharose Fast Flow resin. We pooled the flow-through and buffer exchanged with $50 \mathrm{mM} \mathrm{Na}_{2} \mathrm{HPO}_{4} \mathrm{pH} 8+10 \%$ glycerol using an Amicon filter unit (MWCO $3000 \mathrm{kDa}$ ). The concentration of the cleaved apo-ACP was determined by a Bradford assay.

The conditions for the reactions to generate holo-ACP were: $500 \mu \mathrm{M}$ apo-ACP, $5 \mu \mathrm{M}$ SfP, $5 \mathrm{mM}$ Coenzyme A, and $10 \mathrm{mM} \mathrm{MgCl}_{2}$ in $100 \mathrm{mM} \mathrm{Na}_{2} \mathrm{HPO}_{4} \mathrm{pH}$ 8. The reactions took place in $500 \mathrm{uL}$ aliquots in $1.5 \mathrm{~mL}$ Eppendorf tubes and shaken in a beaker at $37^{\circ} \mathrm{C}$ for $1 \mathrm{~h}$.

We dissolved sodium palmitate in water heated to $65^{\circ} \mathrm{C}$ to a concentration of $100 \mathrm{mM}$. After the holo-ACP reactions were finished, we added palmitate, ATP, and AasS to the reaction mixture, (along with enough buffer to double the reaction volume), to give final concentrations of $5 \mathrm{mM}$ palmitate, $5 \mu \mathrm{M}$ AasS and $10 \mathrm{mM}$ ATP. The reactions incubated overnight $(16-20 \mathrm{~h})$ at $37^{\circ} \mathrm{C}$. Then, we pooled the reactions, purified the palmitoyl-ACP by running the mixture through a gravity column packed with Nickel Sepharose Fast Flow Resin. We buffer exchanged the purified palmitoyl-ACP into $100 \mathrm{mM} \mathrm{Na} \mathrm{HPO}_{4}+10 \%$ glycerol $\mathrm{pH} 8$.

Purification of ATRs. We expressed parental ATRs (A-AAAAAAAA, A-BBBB $\mathrm{BBBB}$, and A-TTTTTTTT) and purified them per the same method as E. coli ACP, except for the buffer exchange step. We buffer exchanged them into $20 \mathrm{mM}$ Tris, $50 \mathrm{mM} \mathrm{NaCl} \mathrm{pH} 7$ using an Amicon filter unit (30,000 kDa MWCO). Then, we added glycerol to the proteins (about $15 \% \mathrm{v} / \mathrm{v}$ for parents 1-3). We expressed ATR83 at $30^{\circ} \mathrm{C}$ rather than $20^{\circ} \mathrm{C}$ but purified it in the same manner, though we added more glycerol to the purified ATR- 83 (final concentration $\sim 50 \% \mathrm{v} / \mathrm{v}$ glycerol). We determined the concentration of the enzymes by Bradford assays.

In vitro enzyme kinetics on palmitoyl-ACP and palmitoyl-CoA. We determined the activity of the above ATRs in a 96 well plate based assay using $5^{\prime} 5$ Dithiobis(2nitrobenzoic acid) or DTNB to monitor the progress of the conversion of palmitoyl-ACP to hexadecanol and free holo-ACP (measuring the absorbance at a wavelength of $412 \mathrm{~nm}$ ). We tested palmitoyl-ACP concentrations up to $40 \mu \mathrm{M}$ (as this concentration should be within the physiological range within cells ${ }^{40}$. Reactions contained $1 \mu \mathrm{M}$ of the respective ATR and $200 \mu \mathrm{M}$ NADPH in $20 \mathrm{mM}$ Tris + $50 \mathrm{mM} \mathrm{NaCl} \mathrm{pH} 7$ and the total reaction volume was $100 \mu \mathrm{L}$. The concentration of DTNB was $250-252 \mu \mathrm{M}$ (the difference is due to slightly different preparations of a NADPH/DTNB master mixes on different dates).

To gauge activity of the ATRs on CoAs in vitro, we carried out reactions using palmitoyl-CoA as a substrate. The in vitro assay used to determine CoA activity was identical to that used for ACP activity above.

Computational docking and analysis of interfacial charge. We used the RosettaDock ${ }^{30,41}$ application to perform local docking simulations to dock a structure of palmitoyl-ACP (from PDB entry 6DFL) to MA-ACR. We did not include the acyl-chain in the docking simulations. We ran 1000 docking simulations and selected a model based on minimizing the total energy and the interface score. Then, using PyMOL, we determined which residues in the model of MAACR were within a $10 \AA$ radius of the ACP molecule. The number of charged residues within that radius was then determined, and the net interface charge was defined as the number of positive residues minus the number of negative residues.

Reporting summary. Further information on research design is available in the Nature Research Reporting Summary linked to this article.

\section{Data availability}

The data supporting the findings of this work are available within the paper and its Supplementary Information files. A reporting summary for this article is available as a Supplementary Information file. Structural data for the following PDB IDs from the protein databank were utilized: 6DFL, 3M1A, 3RKR, 3RIH, 3AFM, 3AFN, 4BMV for structural models. In addition, all enzyme sequence-function data collected in this work is available at the ProtaBank protein engineering database under ID nu9KXbjT4. Source data are provided with this paper.

\section{Code availability}

All code for machine learning, UCB protein sequence optimization, and data analysis is available at the GitHub repository: https://github.com/RomeroLab/ML-Guided-AcylACP-Reductase-Engineering (archived version: https://doi.org/10.5281/zenodo.5259326)

Received: 21 May 2021; Accepted: 1 September 2021; Published online: 05 October 2021

\section{References}

1. Shirmer, A., Rude, M. A., Li, X., Popova, E. \& del Cardayre, S. B. Microbial biosynthesis of alkanes. Sci. (80-.) 329, 559-562 (2010).

2. Hofvander, P., Doan, T. T. P. \& Hamberg, M. A prokaryotic Acyl-CoA reductase performing reduction of fatty Acyl-CoA to fatty alcohol. FEBS Lett. 585, 3538-3543 (2011).

3. Vioque, J. \& Kolattukudy, P. E. Resolution and purification of an aldehydegenerating and an alcohol-generating fatty Acyl-CoA reductase from pea leaves (Pisum SativumL.). Arch. Biochem. Biophys. 340, 64-72 (1997). 
4. Willis, R. M., Wahlen, B. D., Seefeldt, L. C. \& Barney, B. M. Characterization of a fatty Acyl-CoA reductase from marinobacter aquaeolei VT8: a bacterial enzyme catalyzing the reduction of fatty Acyl-CoA to fatty alcohol. Biochemistry 50, 10550-10558 (2011).

5. Metz, J. G. et al. Purification of a jojoba embryo fatty acyl-coenzyme a reductase and expression of Its CDNA in high erucic acid rapeseed. Plant Physiol. 122, 635-644 (2000).

6. Rowland, O. et al. CER4 encodes an alcohol-forming fatty Acyl-Coenzyme A reductase involved in cuticular wax production in arabidopsis. Plant Physiol. 142, 866-877 (2006).

7. Youngquist, J. T. et al. Production of medium chain length fatty alcohols from glucose in Escherichia coli. Metab. Eng. 20, 177-186 (2013).

8. Mehrer, C. R., Incha, M. R., Politz, M. C. \& Pfleger, B. F. Anaerobic production of medium-chain fatty alcohols via a $\beta$-reduction pathway. Metab. Eng. 48, 63-71 (2018).

9. Hernández Lozada, N. J., Simmons, T. R., Xu, K., Jindra, M. A. \& Pfleger, B. F. Production of 1-octanol in escherichia coli by a high flux thioesterase route. Metab. Eng. 61, 352-359 (2020).

10. Opgenorth, P. et al. Lessons from two design-build-test-learn cycles of dodecanol production in escherichia coli aided by machine learning. ACS Synth. Biol. 8, 1337-1351 (2019).

11. Liu, A., Tan, X., Yao, L. \& Lu, X. Fatty alcohol production in engineered E. coli expressing marinobacter fatty Acyl-CoA reductases. Appl. Microbiol. Biotechnol. 97, 7061-7071 (2013).

12. Steen, E. J. et al. Microbial production of fatty-acid-derived fuels and chemicals from plant biomass. Nature 463, 559-562 (2010).

13. Liu, R. et al. Metabolic engineering of fatty Acyl-ACP reductase-dependent pathway to improve fatty alcohol production in Escherichia coli. Metab. Eng. 22, 10-21 (2014)

14. Romero, P. A., Krause, A. \& Arnold, F. H. Navigating the protein fitness landscape with Gaussian processes. Proc. Natl. Acad. Sci. USA. 110 E193-E201 (2013).

15. Yang, K. K., Wu, Z. \& Arnold, F. H. Machine-learning-guided directed evolution for protein engineering. Nat. Methods 687-694 https://doi.org/ 10.1038/s41592-019-0496-6 (2019).

16. Saito, Y. et al. Machine-learning-guided mutagenesis for directed evolution of fluorescent proteins. ACS Synth. Biol. 7, 2014-2022 (2018).

17. Alley, E. C., Khimulya, G., Biswas, S., AlQuraishi, M. \& Church, G. M. Unified rational protein engineering with sequence-based deep representation learning. Nat. Methods https://doi.org/10.1038/s41592-019-0598-1 (2019).

18. Bedbrook, C. N., Yang, K. K., Rice, A. J., Gradinaru, V. \& Arnold, F. H. Machine learning to design integral membrane channelrhodopsins for efficient eukaryotic expression and plasma membrane localization. PLoS Comput. Biol. 13, 1-21 (2017).

19. Liao, J. et al. Engineering proteinase $\mathrm{K}$ using machine learning and synthetic genes. BMC Biotechnol. 7, 1-19 (2007).

20. Fox, R. J. et al. Improving catalytic function by ProSAR-driven enzyme evolution. Nat. Biotechnol. 25, 338-344 (2007).

21. Lennen, R. M., Braden, D. J., West, R. M., Dumesic, J. A. \& Pfleger, B. F. A process for microbial hydrocarbon synthesis: overproduction of fatty acids in Escherichia coli and catalytic conversion to Alkanes. Biotechnol. Bioeng. 106, 193-202 (2010).

22. Voigt, C. A., Martinez, C., Wang, Z. G., Mayo, S. L. \& Arnold, F. H. Protein building blocks preserved by recombination. Nat. Struct. Biol. 9, 553-558 (2002).

23. Silberg, J. J., Endelman, J. B. \& Arnold, F. H. SCHEMA-guided protein recombination. Methods Enzymol. 388, 35-42 (2004).

24. Endelman, J. B., Silberg, J. J., Wang, Z. \& Arnold, F. H. Site-directed protein recombination as a shortest-path problem. Protein Eng. Des. Sel. 17, 589-594 (2004).

25. Srinivas, N., Krause, A., Kakade, S. M. \& Seeger, M. Gaussian process optimization in the bandit setting: no regret and experimental design. IEEE Trans. Inf. Theory 58, 3250-3265 (2009).

26. Auer, P. Using Confidence Bounds for Exploitation-Exploration Trade-offs. J. Mach. Learn. Res. 3, 397-422 (2002).

27. Davis, M. S. \& Cronan, J. Inhibition of Escherichia coli Acetyl Coenzyme A carboxylase by Acyl-Acyl carrier protein. J. Bacteriol. 183, 1499-1503 (2001).

28. Rock, C. O. \& Jackowski, S. Regulation of phospholipid synthesis in Escherichia coli composition of the Acyl-Acyl carrier protein pool in vivo. J. Biol. Chem. 257, 10759-10765 (1982).

29. Sarria, S., Bartholow, T. G., Verga, A., Burkart, M. D. \& Peralta-Yahya, P. Matching protein interfaces for improved medium-chain fatty acid production. ACS Synth. Biol. 7, 1179-1187 (2018).

30. Gray, J. J. et al. Protein-protein docking with simultaneous optimization of rigid-body displacement and side-chain conformations. J. Mol. Biol. 331, 281-299 (2003).
31. Yang, K. K. \& Elliott Robinson, J. Machine learning-guided channelrhodopsin engineering enables minimally invasive optogenetics. Nat. Methods. https:// doi.org/10.1038/s41592-019-0583-8.

32. Jiménez-Osés, G. et al. The role of distant mutations and allosteric regulation on LovD active site dynamics. Nat. Chem. Biol. 10, 431-436 (2014).

33. Pfleger, B. F., Gossing, M. \& Nielsen, J. Metabolic engineering strategies for microbial synthesis of oleochemicals. Metab. Eng. 29, 1-11 (2015).

34. Fiser, A., Kinh Gian Do, R., \& Sali, A. Modeling loops in protein structures Protein Sci. 9, 1753-1773 (2000).

35. Rasmussen, C. E. \& Williams, C. Gaussian processes for machine learning; adaptive computation and machine learning. Vol. 14 (MIT Press: Cambridge, MA, 2006).

36. Desautels, T., Krause, A. \& Burdick, J. W. Parallelizing explorationexploitation tradeoffs in Gaussian Process Bandit Optimization; 2014; Vol. 15.

37. Schindelin, J. et al. Fiji: an open-source platform for biological-image analysis. Nat. Methods. 28, 676-682, (2012).

38. Beld, J., Finzel, K. \& Burkart, M. D. Versatility of Acyl-Acyl carrier protein synthetases. Chem. Biol. 21, 1293-1299 (2014).

39. Néstor, N. et al. Highly active C 8-Acyl-ACP thioesterase variant isolated by a synthetic selection strategy. ACS Synth. Biol. 7, 2205-2215 (2018).

40. Heath, R. J. \& Rock, C. O. Inhibition of $\beta$-ketoacyl-acyl carrier protein synthase III (FabH) by Acyl-Acyl carrier protein in Escherichia coli. J. Biol. Chem. 271, 10996-11000 (1996).

41. Marze, N. A., Roy Burman, S. S., Sheffler, W. \& Gray, J. J. Efficient flexible backbone protein-protein docking for challenging targets. Bioinformatics 34 3461-3469 (2018).

\section{Acknowledgements}

This work was funded by the National Institutes of Health (R35GM119854) and the National Science Foundation (CBET-1703504). J.C.G. is the recipient of a National Institutes of Health Biotechnology Training Program Fellowship (NGIMS

T32GM008349). We would also like to acknowledge Dr. Néstor Hernández Lozada, Dr. Chris Mehrer, and Dr. Mark Politz for helpful discussions regarding assay development, and Bennett Bremer for helpful discussions regarding coding and algorithms.

\section{Author contributions}

J.C.G., B.F.P and P.A.R. conceived the project. J.C.G. performed the experiments with assistance from S.A.F. J.C.G. analyzed the data. J.C.G. and P.A.R. wrote the manuscript with feedback from B.F.P. and S.A.F.

\section{Competing interests}

The authors declare no competing interests.

\section{Additional information}

Supplementary information The online version contains supplementary material available at https://doi.org/10.1038/s41467-021-25831-w.

Correspondence and requests for materials should be addressed to Brian F. Pfleger or Philip A. Romero.

Peer review information Nature Communications thanks David Stuart and other, anonymous, reviewers for their contributions to the peer review of this work.

Reprints and permission information is available at http://www.nature.com/reprints

Publisher's note Springer Nature remains neutral with regard to jurisdictional claims in published maps and institutional affiliations.

\section{(1)}

Open Access This article is licensed under a Creative Commons Attribution 4.0 International License, which permits use, sharing, adaptation, distribution and reproduction in any medium or format, as long as you give appropriate credit to the original author(s) and the source, provide a link to the Creative Commons license, and indicate if changes were made. The images or other third party material in this article are included in the article's Creative Commons license, unless indicated otherwise in a credit line to the material. If material is not included in the article's Creative Commons license and your intended use is not permitted by statutory regulation or exceeds the permitted use, you will need to obtain permission directly from the copyright holder. To view a copy of this license, visit http://creativecommons.org/ licenses/by/4.0/.

(C) The Author(s) 2021 